\title{
The Control Effect of Traffic Information on Traffic Assignment
}

\author{
Junqiang Guo,Bingzhong Ren \\ Department of Information and Electrical Engineering, \\ Shandong University of Science and Technology, Jinan 250031, P.R. China. \\ guojunqiang2008@163.com
}

Keywords: Information; Traffic; Simulation; Intelligent Traffic.

\begin{abstract}
The VISSUM and VISSIM simulation models are setup according to the real road network of Park City, Utah, U.S. The OD matrix is calibrated by the field turning counts. The effects of in-trip real-time information on traffic improvement are studied through microsimulation. Tests have shown that under adaptive traffic control signal, the in-trip real time information has negative feedback effect, and the traffic flows become stable. The information distribution parameters including frequency, delay and proportion of drivers using the information exhibits a window effect. Within the widows, total travel time decreases more than $10 \%$, and total delay decreases more than $20 \%$. The conclusion is that by carefully selecting the distribution parameters of real-time information, the traffic situation of this road network could be improved.
\end{abstract}

\section{Introduction}

Many countries have developed their Intelligent Traffic System(ITS) plan and their own technology standard, such as the United States[1.2], Japan[3] and China. Advanced Traveler Information System(ATIS) is an important part of Intelligent Traffic System. It utilizes communications instruments, like internetwork, television, broadcast, variable information board or in-car computer to provide travelers with information about traffic conditions.

After receiving the information about traffic conditions, travelers may have one of these choices: cancel the travel plan, select another transit mode, another trip route, or change departing time. Thus traffic information may change the traffic requirement and traffic assignment at some extent. If the traffic information can be used correctly, the traffic flow distribution will be changed, and the traffic throng is elevated.

In city road network, most crossroads have traffic signals, and drivers must move according the signal. The basic city traffic elements include driver and car, road network structure, and driver's response to traffic information. Fig. 1 illustrates the feedback effect of traffic information on traffic status. Cars move as a traffic flow under the signal control, the status information feedback to drivers, and drivers respond to the information by rerouting, thus the traffic flow is redistributed.

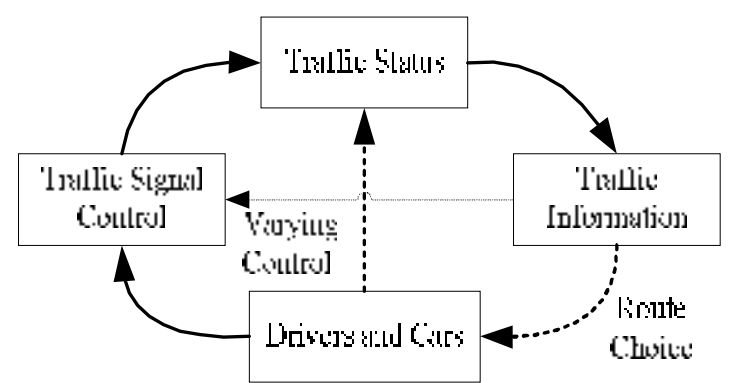

Figure 1. The Feedback of Traffic Information to Traffic Status 
Many researchers have studied the interaction between traffic information and traffic flow. An investigation finds that in all drivers using traffic information, about $34 \%$ drivers changed part route, and $22 \%$ drivers changed their route completely[4]. A simple 4 nodes road network model is setup, and mathematical calculation is taken to analyze the traffic flow distribution. The conclusion is a contradiction between individual and collective goal, that the information of a new road adding in the road network, caused traffic flow redistribution and new traffic throng[5]. An everyday commuting two route choice example is simulated to study the effect of dynamic information on traffic flow stability. The research finds that with the delayed traffic information, the traffic flow becomes instable[6].

The real-time traffic information is available with the modern communications technology. With the feedback of correct traffic information, maybe the traffic flow in a road network could distribute more evenly in space and time, and the traffic throng is elevated in city road.

The development of simulation software and computer technology makes the simulation of real traffic network available. Simulation method is widely used in traffic plan and traffic evaluation[7]. The signal crossroad is analyzed by simulation software[8]. Traffic assignment and signal timing in road network are simulated[9]. A real road network is simulated in which the total travel time and total delay time are studied after traffic control changing from fixed timing to car activated control[10].

\section{Objectives and Hypothesis}

The objective of research is to study the total travel time and total delay time change under traffic control signal responding the traffic flow changing in a city road network when traffic information is broadcasted. We want to know that, providing drivers with in-trip traffic information, whether there is improvement of traffic flow, and how much improvement it brings. The relations between the traffic status and the traffic information broadcast frequency, time delay and the proportion of drivers responding the information are analyzed to find the best traffic information broadcast parameters for alleviating traffic throng in a city road network.

The research hypothesis is based on the concept of traffic equilibrium under signal control, that in mutual consistent flow-control equilibrium, user choices are such that all routes carrying flow have the same cost, and signal-setter's decisions are such that all phases receiving green time sustain the same pressure[11]. In such circumstance, the administer change the signals according to traffic status, and the drivers select their routes and departure time according to the traffic information received.

Suppose that driver's route choice standard is minimum cost, and the cost in this research is travel time. Radom route choice model is taken, most drivers chose the minimum travel time route, and there are some drivers select the others routes. When detour route exceeds 2.5 times the shortest route, the driver tends to maintain the selected route.

\section{Simulation Model}

The PTV Vision software VISUM 11.0 and VISSIM 5.0 is used in this research. VISUM is a travel demand modeling software, and is used for Origin-Demand(OD) matrix correction in this paper. VISSIM is a microscopic traffic simulator, and is used to emulate realistic traffic system here.

VISSUM and VISSIM software are widely used as a research tool. The OD matrix correction module in VISSUM is studied, and proved it a reliable matrix correction tool[12]. The reliability of VISSIM simulation is compared in different circumstances, and the conclusion is that it is a reliable micro simulation tool[13].

Park City road network is used as a research network. There are 14 signal crossroads, which uses Adaptive Traffic Controls, Split Cycle Offset Optimization Technique (SCOOT), as traffic control signal. According to the road network, a VISSUM simulation model is setup as Fig. 2, in which there are 376 links, 54 OD pairs, 1082 turns and 125 detectors. A VISSIM simulation model is setup as Fig. 3, in which there are 108 parking lots, 182 signal heads, 226 detectors, 1594 links/connectors, 260 data collecting points, and 220 nodes. 


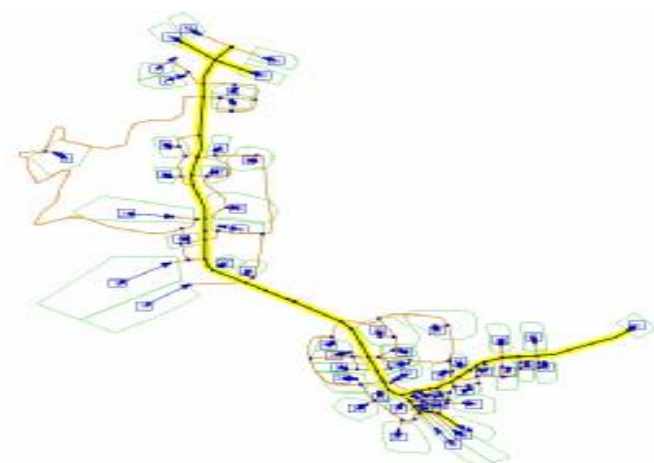

Figure 2. VISSUM Simulation Model

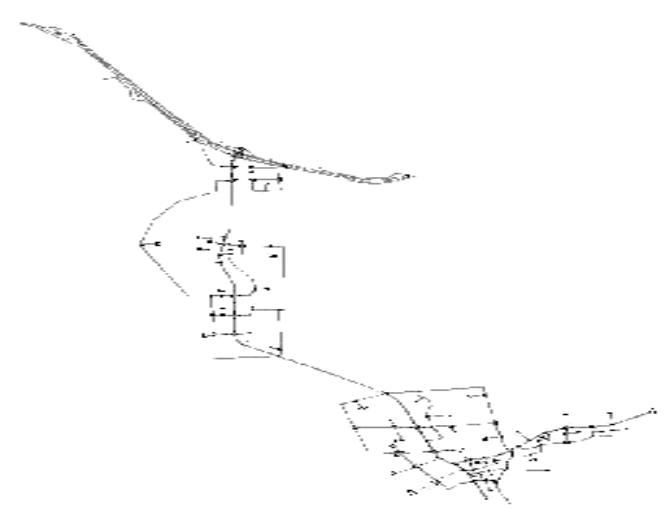

Figure 3. VISSIM Simulation Model

The field count data of turned cars are used to correct OD matrix. 125 data is collected at the intersections. After 10 iterations OD matrix-correction and assignment in VISUM simulation model, an OD matrix is obtained, with $\mathrm{R}^{2}$ of 0.98 for modeled data vs. field counts. Fig. 4 and Fig. 5 depict the scattered plot for modeled versus count data for initial and calibrated OD matrices.

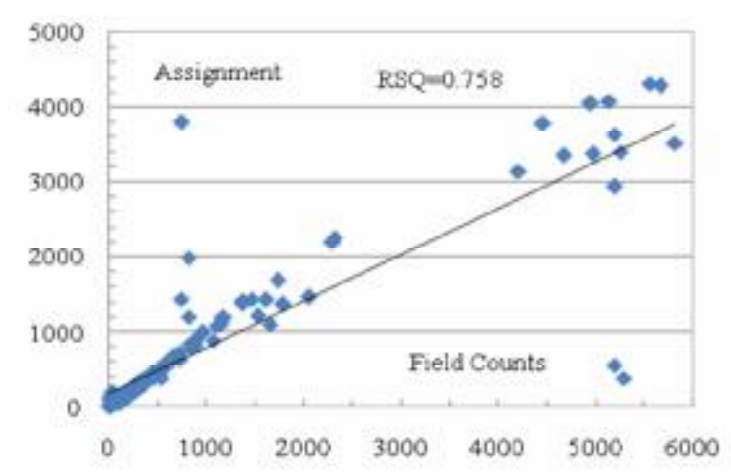

Figure 4. Modeled vs. Field Counts From Initial OD Matrix 


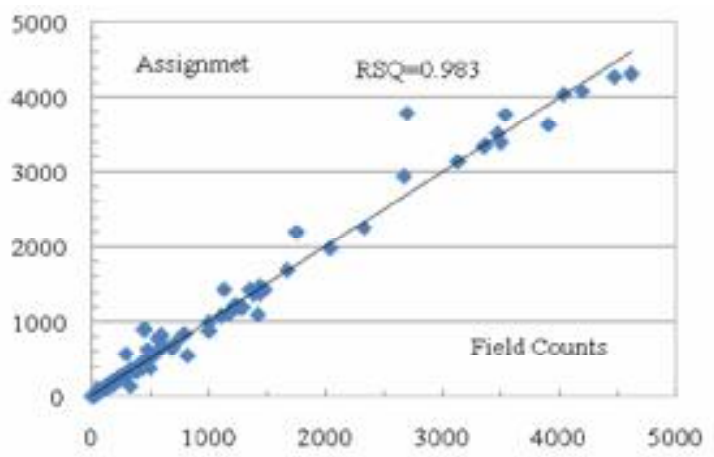

Figure 5. Modeled vs. Field Counts From Calibrated OD Matrix

The traffic information collecting and processing need time and the information transmission to driver need time also. Moreover, drivers need time to make rerouting decision after received information, and to move cars forming traffic flow. The traffic flow has a time delay with traffic information. The time delay is equivalent to delayed broadcasting traffic information in the simulation. There are three information broadcasting parameters, frequency, delay and proportion of drivers using the information. Nine test scenarios are designed as Table 1.

Table 1 Test Scenarios

\begin{tabular}{|c|c|c|c|}
\hline $\begin{array}{c}\text { Test } \\
\text { scenarios }\end{array}$ & $\begin{array}{c}\text { Frequency } \\
\text { (Seconds) }\end{array}$ & $\begin{array}{c}\text { Delay } \\
\text { (Seconds) }\end{array}$ & $\begin{array}{c}\text { Proportion } \\
\text { of Drivers }\end{array}$ \\
\hline 0 & no & no & no \\
\hline 1 & 1200 & 10 & $\% 0 \sim 100 \%$ \\
\hline 2 & 120 & 10 & $\% 0 \sim 100 \%$ \\
\hline 3 & $10 \sim 3600$ & 10 & $80 \%$ \\
\hline 4 & $10 \sim 3600$ & 10 & $20 \%$ \\
\hline 5 & 120 & $5 \sim 120$ & $20 \%$ \\
\hline 6 & 1200 & $10 \sim 1200$ & $20 \%$ \\
\hline 7 & 120 & $5 \sim 120$ & $80 \%$ \\
\hline 8 & 1200 & $10 \sim 1200$ & $80 \%$ \\
\hline
\end{tabular}

\section{Results and Discussion}

From the traffic administration point of view, the minimum total travel time and the minimum total delay time are expected. Each simulation of test scenario in Table 1 is run 100 times. From test scenario 0 to test scenario 8 , total travel time and total delay time of the road network are calculated. The average total travel time and total delay time from test scenario 1 to test scenario 8 are compared with the values of test scenario 0 . The improvement of total travel time and total delay time is depicted in Fig. 6 to Fig. 9.

Taking total travel time decreasing $10 \%$ and total delay time reducing $20 \%$ as a standard, the frequency, delay or proportion of drivers has window effect with the tow other parameters fixed.

It is can be seen in Figure 6 to Figure 8 that total travel time and total delay time are increasing at some points, and the maximum value is $7.7 \%$.

For the road network studied, the traffic information has a control effect on traffic assignment. Under SCOOT Adaptive Traffic Controls, the real-time information makes traffic flow more stable and improves both total travel time and total delay time if the information broadcasting parameters were properly selected. 


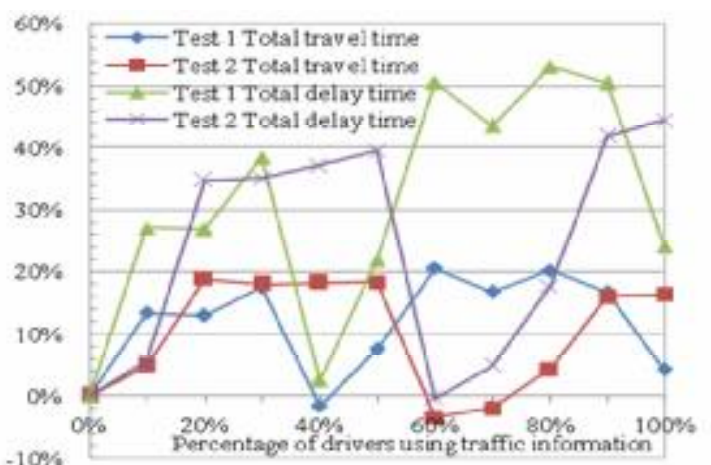

Figure 6. Total Travel Time and Total Delay Improvement of Test Scenario 1 and 2

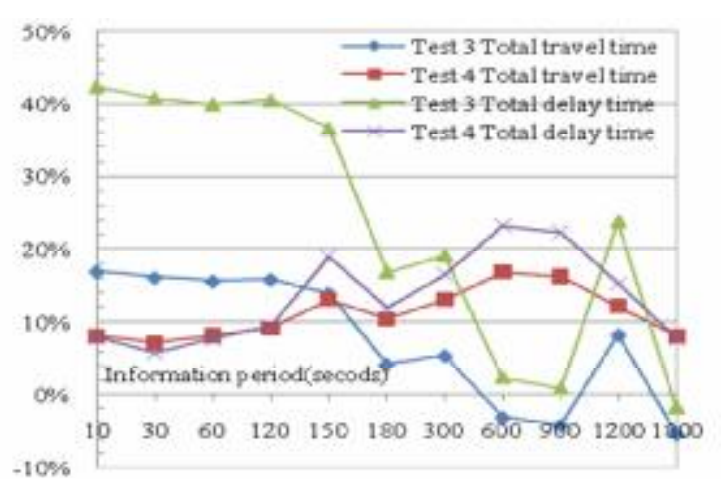

Figure 7. Total Travel Time and Total Delay Improvement of Test Scenario 3 and 4

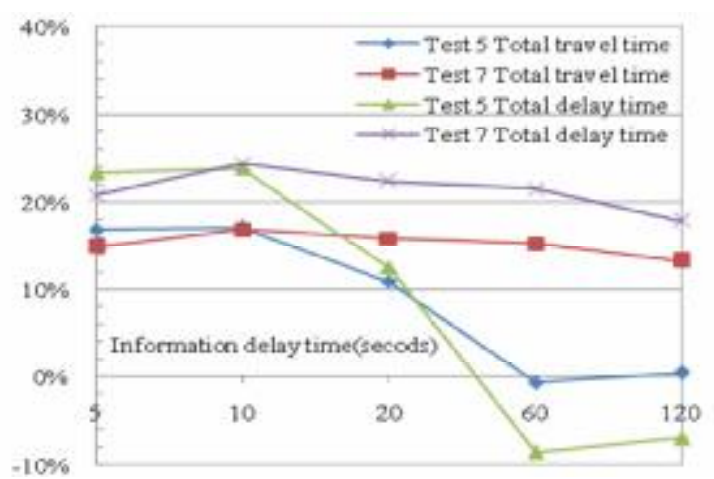

Figure 8. Total Travel Time and Total Delay Improvement of Test Scenario 5 and 7

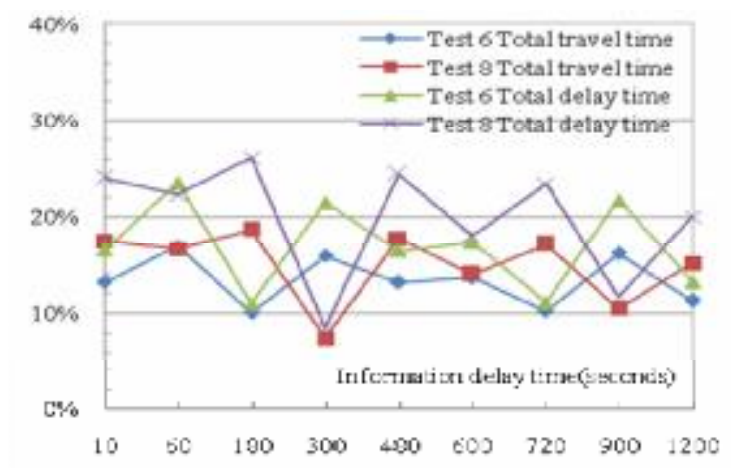

Figure 9. Total Travel Time and Total Delay Improvement of Test Scenario 6 and 8 
The research is done in Utah Traffic Lab, University of Utah, the United States. Thanks Doctor Peter T. Martin, Muhammad Farhan and other staffs for their helps.

\section{References}

[1]Intelligent Transportation Society of America. National Intelligent Transportation Systems Program Plan, VOLUME I, (1995) http://www.itsdocs.fhwa.dot.gov/jpodocs/repts_pr/3827.pdf.

[2]Intelligent Transportation Society of America. National Intelligent Transportation Systems Program Plan, VOLUME II, (1995) http://www.itsdocs.fhwa.dot.gov/jpodocs/repts pr/3786.pdf.

[3]Tadashi Okutani. Policy for Cooperative ITS in Japanese Smartway Project, (2008) http://www.its.go.jp/ITS/conf/2008/ES07.pdf.

[4]Mehndiratta S R, Kemp M A, Lappin J E, Nierenberg E. Likely Users of Advanced Traveler Information Systems, Washington DC: Transportation Research Record 1739, (2000)15-24.

[5]Dietrich Braess. On a Paradox of Traffic Planning, Transportation Science, 39(4) (2005) 446-450.

[6]Joachim Wahle, Ana Lucia C, et al. Decision dynamics in a traffic scenario, Physica A 287, 15(6) (2000)669-681.

[7]Bloomberg L, Dale J. Comparison of the VISSIM and CORSIM traffic simulation models on a congested network, Transportation Research Record 1727, (2000) 52-60.

[8]Yaldi G. and Yue W. The use of Cube Dynasim and aaSIDRA in analyzing a signalized intersection, 29th Australian Transport Research Forum, Australia, (2006) 639-652.

[9]Abdelfatah A S, Mahmassani H S. System Optimal Time-Dependent Path Assignment and Signal Timing in Traffic Network, Transportation Research Record 1645, (1998) 185-193.

[10]Farhan M, Stevanovic A, Martin P. A Practical perspective on the benefits of Combined Traffic Assignment and Control Method, Proceedings of 89th Annual Transportation Research Board Conference, Washington, (2010) 121-125.

[11]Smith M J, van Vuren T. Traffic Equilibrium with Responsive Traffic Control, Transportation Science, 27(2) (1993) 118-132.

[12]Jha M, Gopalan G, Garms A, Mahanti, et al. Development and calibration of large-Scale microscopic traffic simulation model, Transportation research Record 1876, (2005) 121-131.

[13]Fellendorf $\mathrm{M}$ and Vortisch P. Validation of the microscopic traffic flow model VISSIM in different real-world situations, 80th Meeting of the Transportation Research Board, Washington DC, (2001) 2014-2035. 